\title{
A STUDY OF THE FORCES PRODUCTIVE OF GASTRO- OESOPHAGEAL REGURGITATION AND HERNIATION THROUGH THE DIAPHRAGMATIC HIATUS
}

\author{
BY \\ PAUL MARCHAND \\ From the Department of Surgery, University of the Witwatersrand, Johannesburg
}

(RECEIVED FOR PUBLICATION MARCH 20, 1957)

A great wealth of literature on the subject of gastro-oesophageal regurgitation and hiatus hernia has accumulated in recent years. The two conditions are closely linked and frequently, but by no means invariably, associated (Marchand, 1955). The work of Allison (1948 and 1951), Barrett (1954), Creamer (1955), Donnelly (1953), and many others has done much to clarify the subject which, before the association of reflux dyspepsia and hiatus hernia, was ill understood. Important original work on the anatomical relationship of the cardia and diaphragm and on the structural changes which accompany the development of a hernia has recently been reported. The writings of Allison (1951) have pointed the way, whilst those of Barrett (1954), Collis, Kelly, and Wiley (1954), Collis, Satchwell, and Abrams (1954), Harrington (1952), Lerche (1950), and Peters (1955) have done much to advance understanding of the condition. In the main, interest has been centred upon the clinical features of hiatus hernia and upon the anatomical changes which occurunderstandably enough, for the diagnosis and surgical management of the condition must depend on a sound knowledge of these. The question of the forces which tend to extrude the stomach and its contents into the chest and oesophagus has received scant attention, a surprising omission, for without such a force no hernia could develop and regurgitation would never occur. It is the object of this paper to report certain experiments which have been performed to measure and record the pressures which are exerted upon the diaphragm and cardia.

\section{EXPERIMENTAL Method}

In the past, most reports of intraperitoneal pressures were based upon measurements recorded within the lumen of the gut with hydrostatic balloons (Wilson and Irving, 1931 ; Lam, 1939). Quigley (1944) realized that this method was open to grave criticism when used to determine absolute pressures, for the elastic balloon exerts its own influence upon the conducting system. He therefore adopted the "open tube" method for his experiments in animals, inserting a catheter of $3 \mathrm{~mm}$. bore directly into the lumen of the gut. Dornhorst and Leathart (1952) have applied this technique, with modifications, to studies in man. They introduce a polythene catheter of $\frac{1}{2} \mathrm{~mm}$. internal bore into the oesophagus or stomach via the nasopharynx. They use a thin tube so as not to stimulate intestinal contractions. With this method they have shown that the intra-oesophageal pressure accurately reflects the intrapleural pressure. This technique has been followed without modification in the present experiments. A Sanborn strain-gauge manometer has been used and pressures have been calibrated in centimetres of water with a standard water manometer. Only a single side-hole near the tip of the catheter has been made so that the pressures would be recorded at a single spot. (Unless otherwise stated, the experiments have been done on human subjects, and I am grateful to the students of our medical school who volunteered to be the subjects.)

\section{The Movements of the Diaphragm}

The diaphragm descends during inspiration, producing a negative pressure within the pleural cavity. The greatest excursion occurs at the dome, which may move as much as 4 in. $(9 \mathrm{~cm}$.) in an efficient abdominal breather (Appleton, Hamilton, and Simon, 1946). However, the extent of diaphragmatic excursion depends on many factors such as the build of the patient, body posture, and the volume of the lungs and abdominal contents (Wade and Gilson, 1951). Fluoroscopic methods only show a silhouette of the diaphragm and the position of the oesophageal hiatus cannot be accurately visualized. Movements of the barium-outlined gastro-oesophageal junction do not necessarily reflect the position of the hiatus. Limited movement of the terminal oesophagus can occur within the hiatus so that it may slide upwards when the hiatus moves downwards. That the hiatus does in fact descend on inspiration is easily proved. 
A polythene catheter, with its tip marked with a lead pellet, is passed into the stomach and connected to an electromanometer. One knows that the catheter tip lies below the diaphragm by the positive inspiratory deflection of the pressure tracing. If the catheter is slowly withdrawn during shallow respiration the curve will suddenly deflect to negative and typical intrapleural pressure curves will be recorded. The point of transition marks hiatal level and not the gastro-oesophageal junction as was proved in a case of fixed hiatus hernia where the sudden change occurred at a level lower than that of the cardia. If the catheter is now advanced a short distance into the stomach and its position fixed while the subject takes a deep breath, the initial inspiratory curve will be positive but suddenly it will deflect negatively $y_{0}^{\stackrel{\vec{F}}{+}}$ (Fig. 1). The further the catheter tip advances: the later the negative deflection occurs, until a point is reached when the pressure remains posi- $\bar{D}$ tive throughout the phase of inspiration (Fig. 2) The biphasic wave is produced because the record- ing point lies initially in the stomach below hiatus $\vec{\circ}$ level ; but, as the diaphragm descends, the hiatus slips past it and a supradiaphragmatic pressure is recorded. The distance that the catheter is advanced while registering a biphasic wave is measure of hiatal excursion. This experiment has been done on six medical students and the hiatushas been found to move an average of $2 \mathrm{cmc}$ downwards during deep inspiration.

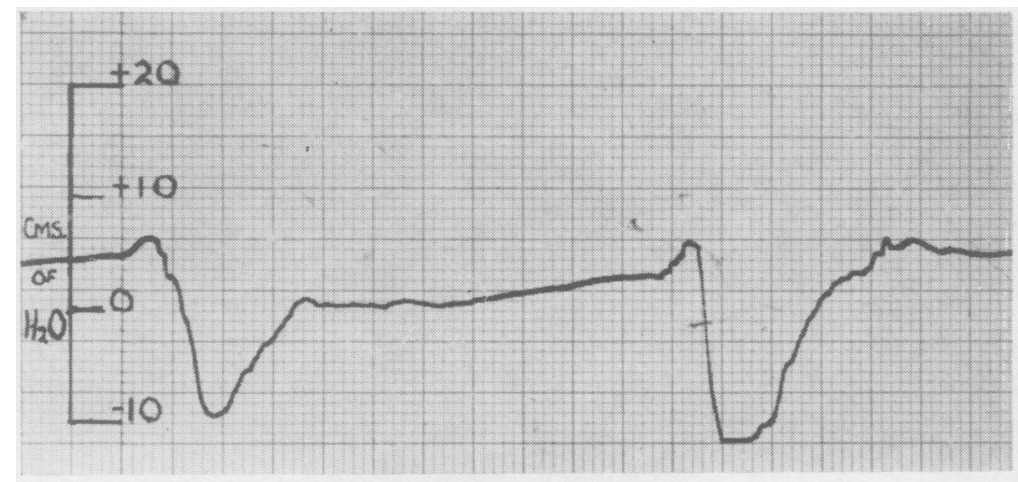

Paper Speed $10 \mathrm{~mm}$./second

Fig. 1.-Pressure tracing taken during inspiration with the catheter tip lying at hiatus level. A constant situation of the tip was maintained by fixing the head and trunk and anchoring the catheter to the patient's nostril. Note the initial positive deflection which denotes a subphrenic situation followed by the sudden negative deflection as the hiatus slips below the recording point.

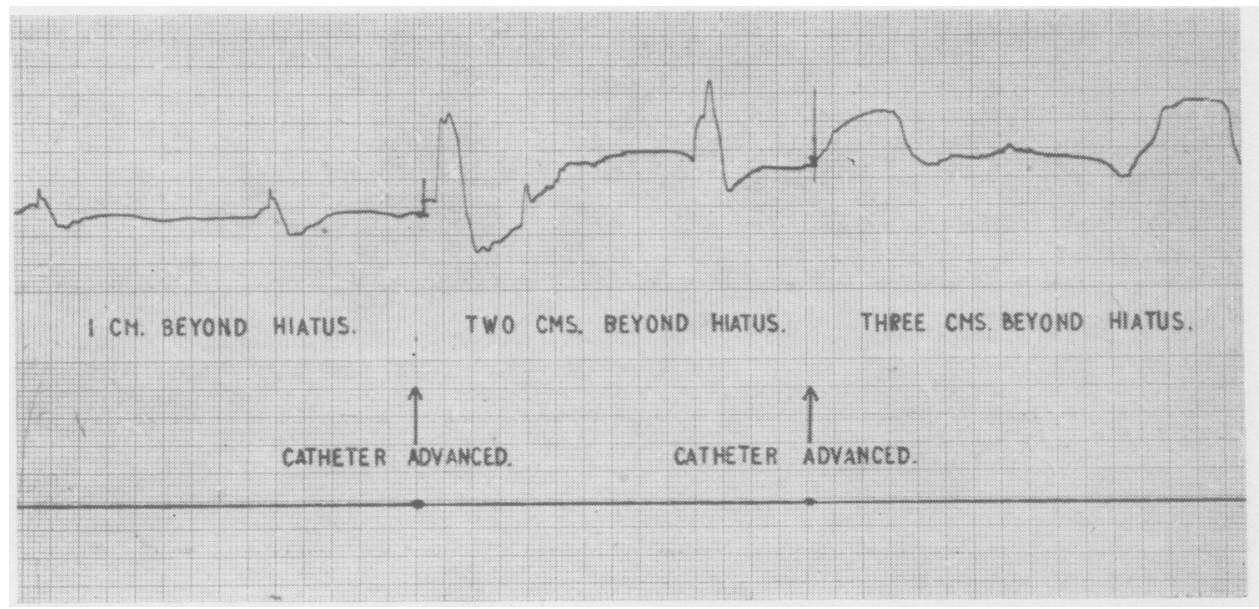

FIG. 2.-Deep inspiratory tracings recorded after the catheter tip had been advanced 1, 2, and $3 \mathrm{~cm}$. beyond hiatus level. 


\section{The Extrinsic Forces Acting on the DiAPHRAGM}

The total separation of the thoracic cavity from the abdomen is a distinctive mammalian characteristic. The diaphragm, acting as a barrier, prevents the dissipation of the negative intrapleural pressure while its own activity plays a major part in causing changes of pressure within the abdomen and thorax.

If one considers a closed space of fixed capacity divided by a mobile septum, then descent of the septum will lower the pressure above it whilst the thrust acting below will increase progressively. Should a localized weakness be present in the septum the affected area would tend to bulge into the upper compartment as the septum descends. This illustrates the possible effect that the diaphragmatic contraction may have upon its own inherent weaknesses. The analogy cannot, however, be carried very far. In the first place the capacities of the abdominal and thoracic cavities are not rigidly fixed and the mobility of their walls may modify the effect of diaphragmatic descent. Secondly, contraction of the powerful abdominal muscles is also capable of increasing the intraperitoneal pressure. The third variable is that of body posture, which has a decided influence upon the abdominal pressure. The peritoneal cavity is largely occupied by mobile organs whose positions are influenced by gravity. With the body erect the abdominal viscera tend to "fall away" from the diaphragm, and, because actual separation is not possible, a negative pressure effect is produced within the upper abdomen. In the "pelvic-high" position the effect is reversed. An attempt has been made in the following experiments to confirm these theoretical observations.

\section{Intragastric Pressure as a Measure of General Intraperitoneal Pressure in THE HORIZONTAL POSITION}

One catheter was passed through the subject's mouth and a second into the rectum through a sigmoidoscope. Simultaneous tracings were obtained with a twin-channel direct-writing recorder. The zero reference plane was taken to be the midpoint between the xiphisternum and the table surface with the subject supine.

Fig. 3A shows that when the subject strains forcefully the rise in pressure within the stomach is closely paralleled by that within the rectum.

In the second subject gastric and vesical pressures showed identical deflections during straining (Fig. 3B). In a patient with ascites, a needle was inserted into the peritoneal cavity and simultaneous intravesical and intraperitoneal pressures were recorded. Again the pressure tracings are closely similar (Fig. 4).

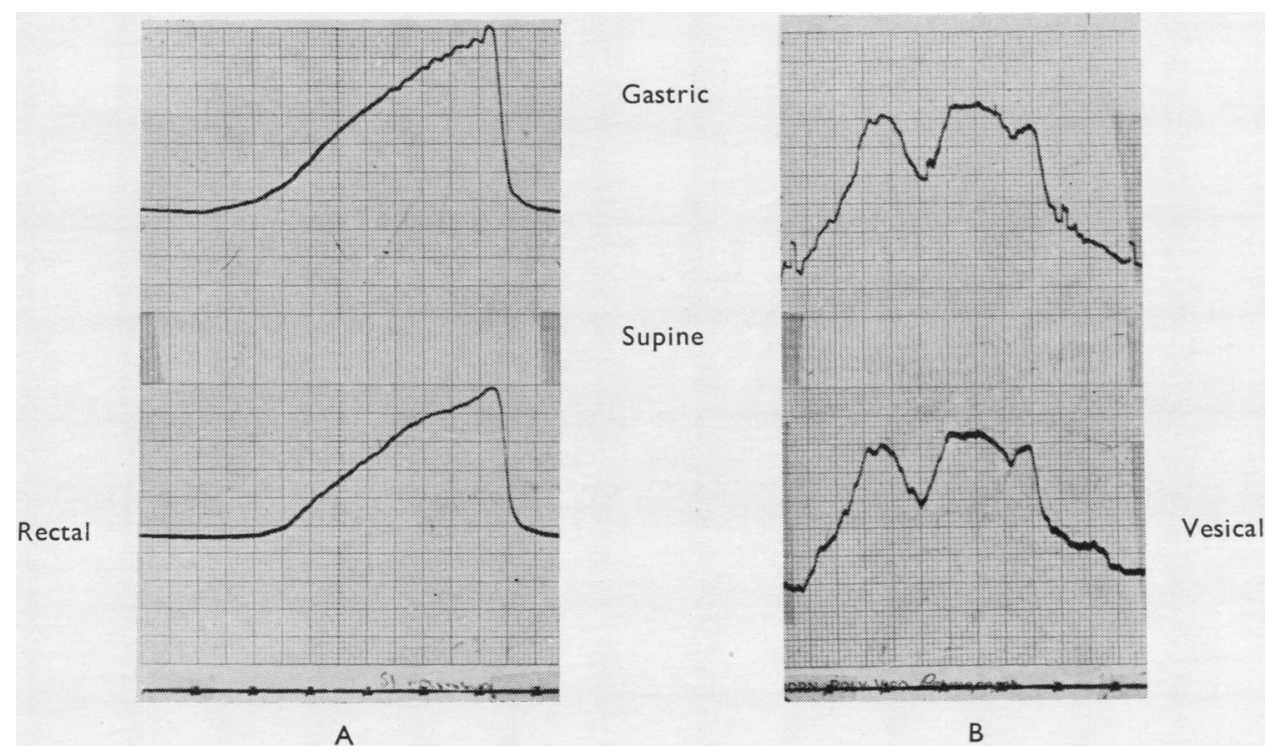

FIG. 3.-Simultaneous pressure records (A) within stomach and rectum, (B) within stomach and bladder. The shape and amplitude of the tracings are closely paralleled in both experiments. 


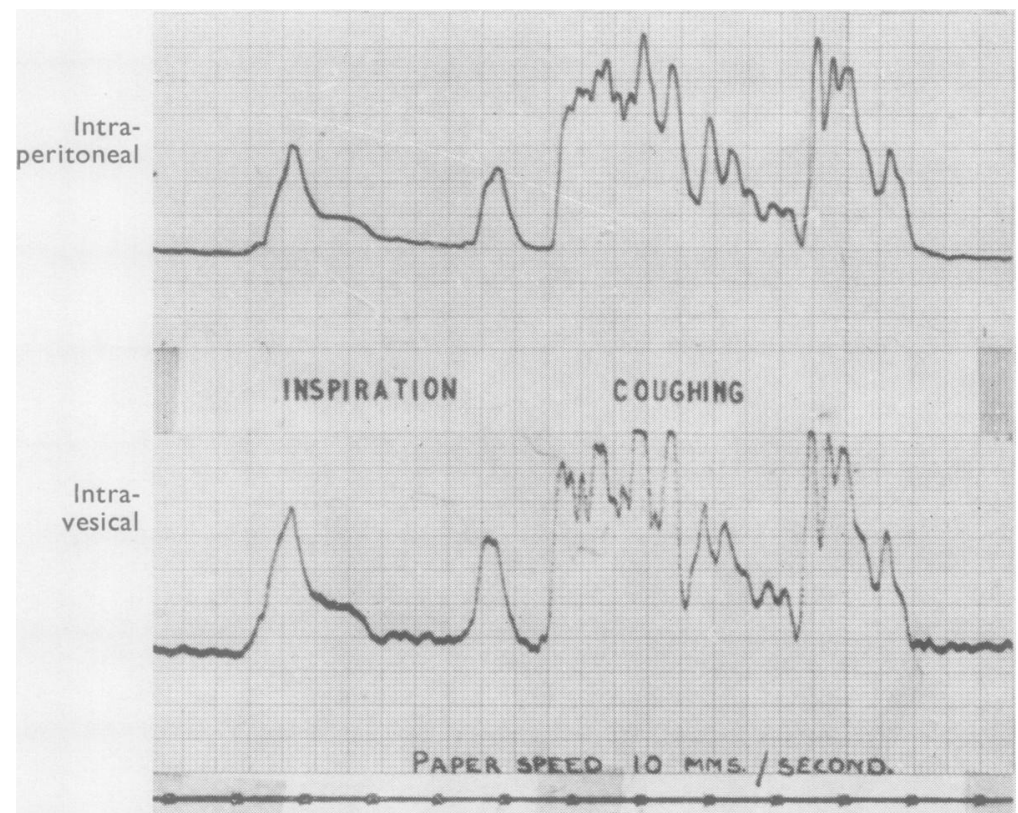

Fig. 4.-Simultaneous intraperitoneal and intravesical pressures recorded during deep inspiration and coughing in a patient with ascites.
These experiments confirm that with the stomach at rest the intragastric pressure is valid as a measure of pressure throughout the hollow viscera of the abdomen and that these reflect the general intraperitoneal pressure with the subject in the supine position. When peristalsis occurs the pressure effect is superimposed upon the graph of the intraperitoneal pressure and is easily recognizable as such.

\section{InTRaPeritoneal Pressures in the EREct Subject}

In this experiment the catheter tip was manœuvred so as to lie in the stomach about 4 $\mathrm{cm}$. beyond the cardia. A second catheter was then introduced into the rectum. It was considered that the recordings would be better comparable if they were taken immediately consecutively using the same pressure and recording channel. Accordingly the two catheters were connected by a two-way tap to the manometer which rested upon a movable shelf. The subject stood erect and the manometer was adjusted to lie at the level of the xiphisternum. Intragastric pressures were recorded during quiet respiration. The manometer level was then lowered to the plane of the upper edge of the pubic symphysis and the two-way tap was adjusted so that the rectal pressure would be recorded. The experiment was repeated with the subje $\overrightarrow{\overline{\vec{t}}}$ tilted head downwards at ato angle of $45^{\circ}$ from the hore zontal.

Allowing for some errof in the adjustment betweep catheter points and manos meter levels (zero reference points), it is quite obvious that gravity does influen the intraperitoneal prest sures (Fig. 5). The differ ence in the mean pressures between the two levels with the body erect was 18 cne of water, and with the trund inclined downwards at $45^{\ni}$ the difference was $8 \frac{1}{2} \mathrm{~cm}$. cs

The Pleuro-peritoneal Pressure Gradient

Simultaneous supine oeso phageal and gastric pre $\vec{\varnothing}$ sures have been recorded five medical students thig had fasted for six hodre Pressures were taken whilst the subjects performed the following actions: (a) Graduated increase the depth of respiration; $(b)$ coughing; $(c)+$ th Valsalva test ; $(d)$ lifting both heels off the groung with legs extended.

RESPIRATION.-During quiet respiration in the supine position, the average gastric pressure flue tuates from $+2 \mathrm{~cm}$. of water on expiration $+10 \mathrm{~cm}$. of water on inspiration, whilst the corre sponding pleural pressures are $-5 \mathrm{~cm}$. of wat and $-10 \mathrm{~cm}$. of water (Fig. 6A). Thus in the supine position the average pressure gradie during quiet breathing fluctuates from 7 to $\dot{z}$ $\mathrm{cm}$. of water. With progressively deepenin respiration the intrapleural inspiratory pressure becomes increasingly negative whereas the intraperitoneal pressures become increasingly positi (Fig. 6B). When the subject inspires with max mum effort the gastric pressure may rise to $50 \mathrm{cn} \Phi$ of water (Fig. 6C) and the pleuro-peritoneab gradient may exceed $100 \mathrm{~cm}$. of water. This is considerable stress which the diaphragm has to resist.

Coughing.-Fig. 7 illustrates the effect of coughing on the intrarectal and intra-oesophageat pressures. Considerable pressures are momen tarily generated during a cough, and for purpose of graphic reproduction it is often necessary to 


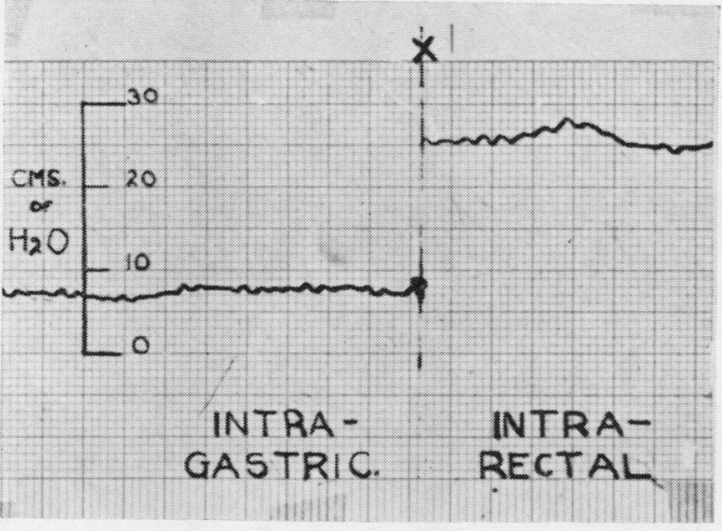

A

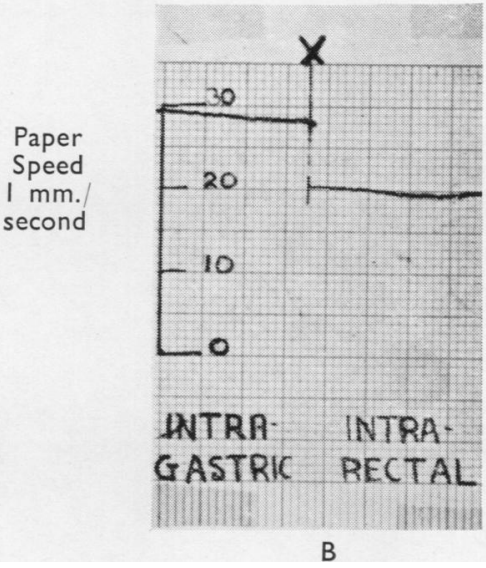

B

FIG. 5.-Immediately consecutive intragastric and intrarectal pressures recorded (A) with the subject erect and (B) with the subject tilted head downwards at an angle of $45^{\circ}$.

adjust the sensitivity of the recorder so that the stylus is not forced off the paper. The intrarectal record shown as Fig. 7 has been so damped that the respiratory excursions are scarcely visible and consequently the absolute pressures generated are not comparable. The figure serves to illustrate that both intrapleural and intraperitoneal pressures become markedly positive during coughing so that the actual pleuro-peritoneal pressure gradient is not greatly increased.

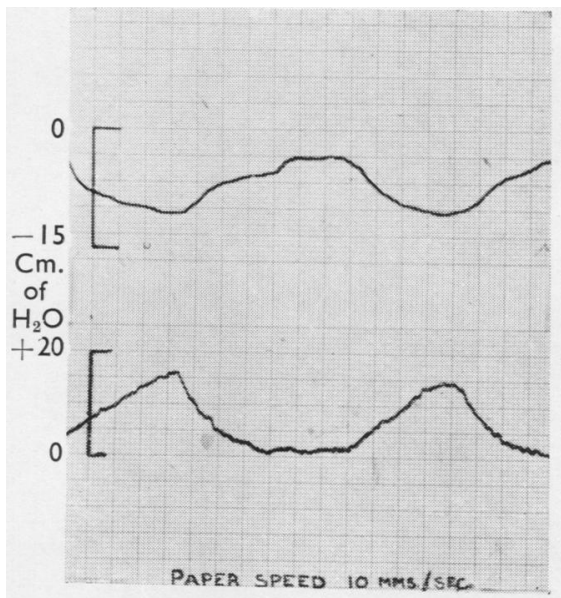

A

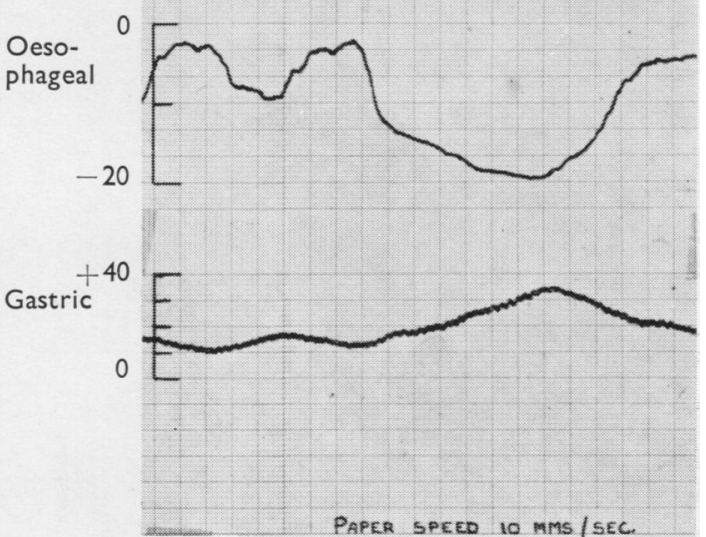

B

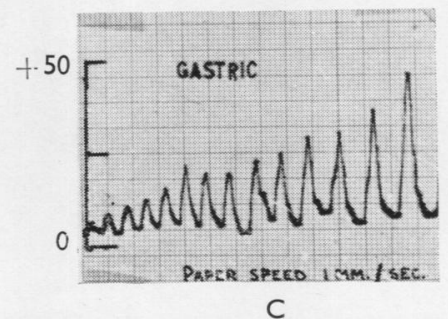

FIG. 6.-Pressure tracings recording the changes in the pleuro-peritoneal pressure gradient during (A) quiet respiration, (B) deep inspiration, (C) increasing depth of respiration (gastric pressure only). 


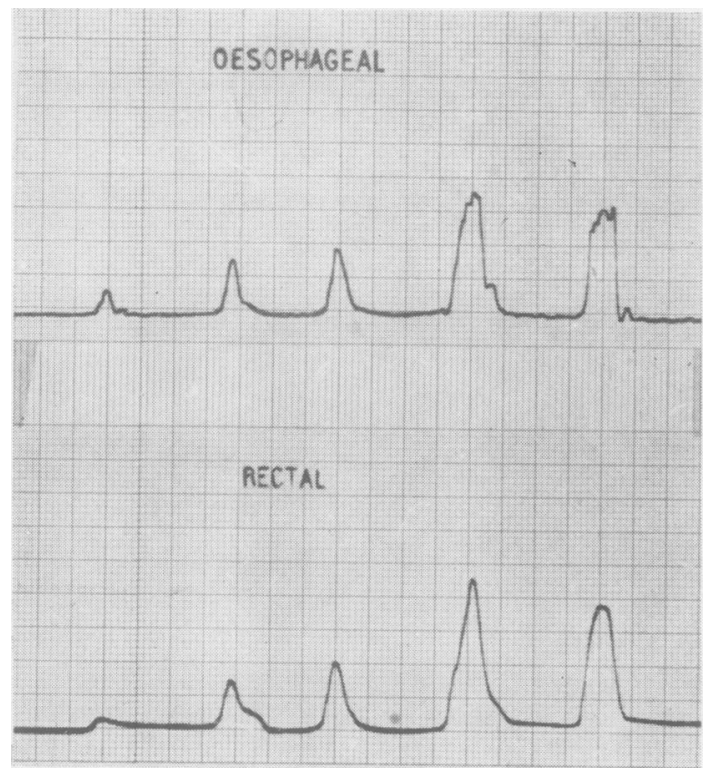

FIG. 7.-Simultaneous oesophageal and rectal pressures taken while the subject gradually increased the force of isolated coughs.
The Valsalva Maneeuvre.-In a previouse्? paper (Marchand, 1955) it was shown that botho intraperitoneal and intrapleural pressures rise during the performance of the Valsalva test. The $\frac{\bar{\omega}}{5}$ pleuro-peritoneal pressure gradient remains con- $\mathbb{\Phi}$ stant at about $10 \mathrm{~cm}$. of water during the earlyo effort, but, with forceful straining against the closed glottis, the intraperitoneal pressure sud-. denly outstrips the intrapleural pressure. This $\overrightarrow{\vec{H}}$ sudden increase in the pressure gradient across theo diaphragm is thought to be due to the forcefulf abdominal muscle contraction exercising a greater ${ }_{-}^{x}$ effect upon the abdominal than upon the thoracic? pressures.

Simultaneous intra-oesophageal and intragastric $\overrightarrow{0}$ pressures, recorded with an electromanometer,응 confirm these results. Thus straining, as at a stool, does increase the pleuro-peritoneal pressurecs gradient, though not to a degree that it does be $\frac{\mathbb{D}}{0}$ tween the peritoneum and the exterior. During $\mathbb{\mathbb { Q }}$ the Valsalva manœuvre intraperitoneal pressures $\overline{0}$ of more than $100 \mathrm{~cm}$. of water may be produced. $\stackrel{\Phi}{~}$ This pressure acts directly upon the external $\vec{c}$ hernial sites, but the thrust on the diaphragm is
viso Prmapapet

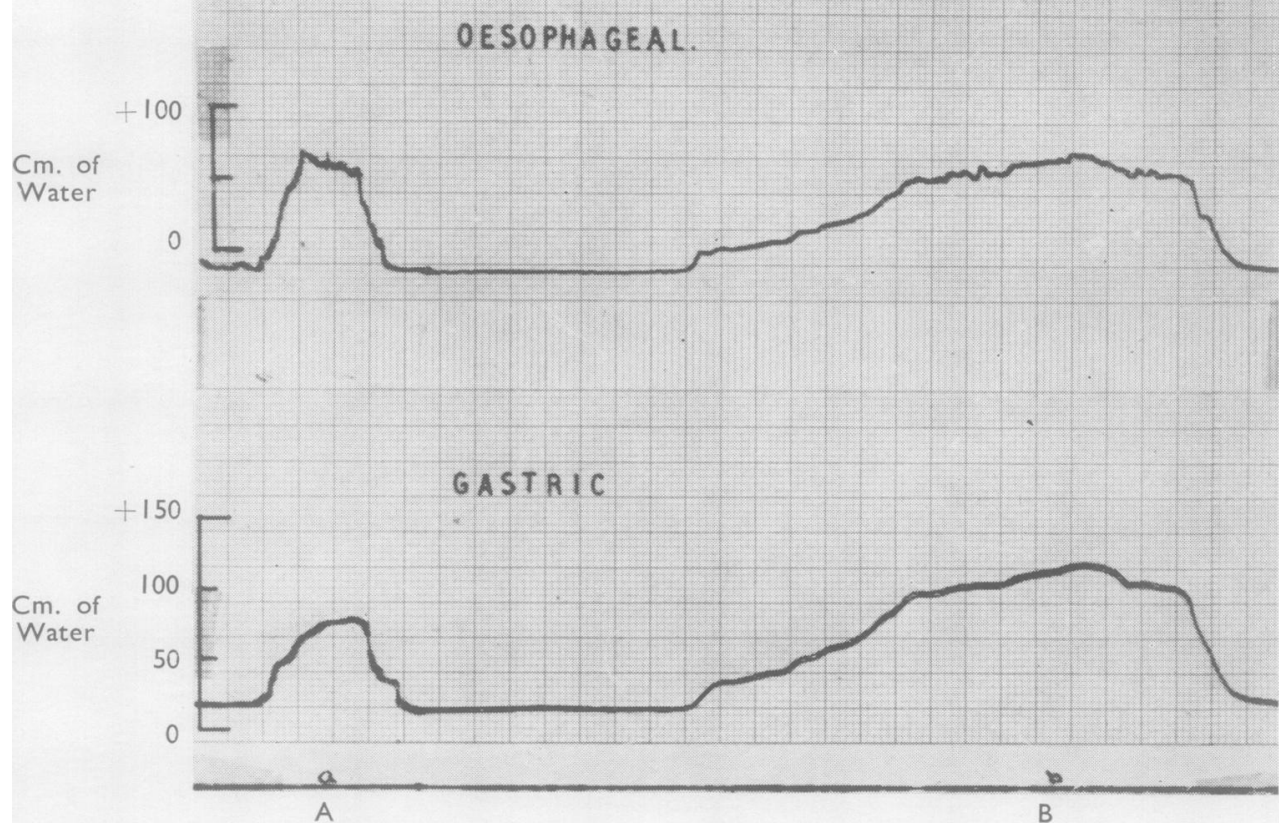

Fig. 8.-Simultaneous intra-oesophageal and intragastric pressures recorded while the subject performed the Valsalva test: (A) straining moderately when the pleuro-peritoneal pressure gradient is $15 \mathrm{~cm}$. of water; (B) straining with maximum effort when the gradient is $45 \mathrm{~cm}$. of water. 
cushioned by the simultaneous increase of the thoracic pressure. During the Valsalva test a pleuro-peritoneal gradient of about $30 \mathrm{~cm}$. of water is produced (Fig. 8) which is considerably less than that generated by deep inspiration.

Tensing Abdominal Muscles.-Straight leg raising increases the intraperitoneal pressure considerably $(120 \mathrm{~cm}$. of water $)$, but, as with the Valsalva test, the subject strains against the closed glottis and the thoracic pressure rises simultaneously. Here also the abdominal pressure rises more rapidly than the thoracic pressure so that the pleuro-peritoneal gradient does increase.

EXTRANEOUS INFLUENCES ON IN IRAPERITONEAL Pressures.-In one experiment the effect of external compression upon the abdominal pressure was recorded. An abdominal corset incorporating an inflatable balloon centred over the umbilicus was used (Marchand, 1952). The balloon pressure was progressively raised by $20 \mathrm{~mm}$. of mercury with the recording paper running at a speed of $1 \mathrm{~mm}$. per second. From a mean level of $6 \mathrm{~cm}$. of water the abdominal pressure rose progressively as the external compression was increased and with an external pressure of 120 $\mathrm{mm}$. of mercury the intraperitoneal pressure registered approximately $30 \mathrm{~cm}$. of water (Fig. 9A).

The effect of suddenly tightening a corset was studied in a separate experiment and pleural and peritoneal pressures were recorded. The corset embraced the whole abdomen and was tightened by two assistants suddenly pulling on the ends and hooking them together. This was done so as to simulate conditions which a person suffers when wearing that garment. The subject lay relaxed and prone and the hitching process was done over the back while the recording was being made (Fig. 9B). The effect of this manœuvre was to increase the mean intraperitoneal pressures which rose on inspiration from a level of $10 \mathrm{~cm}$. of water to one of $24 \mathrm{~cm}$. of water. The effect on the intrapleural pressure was slight.
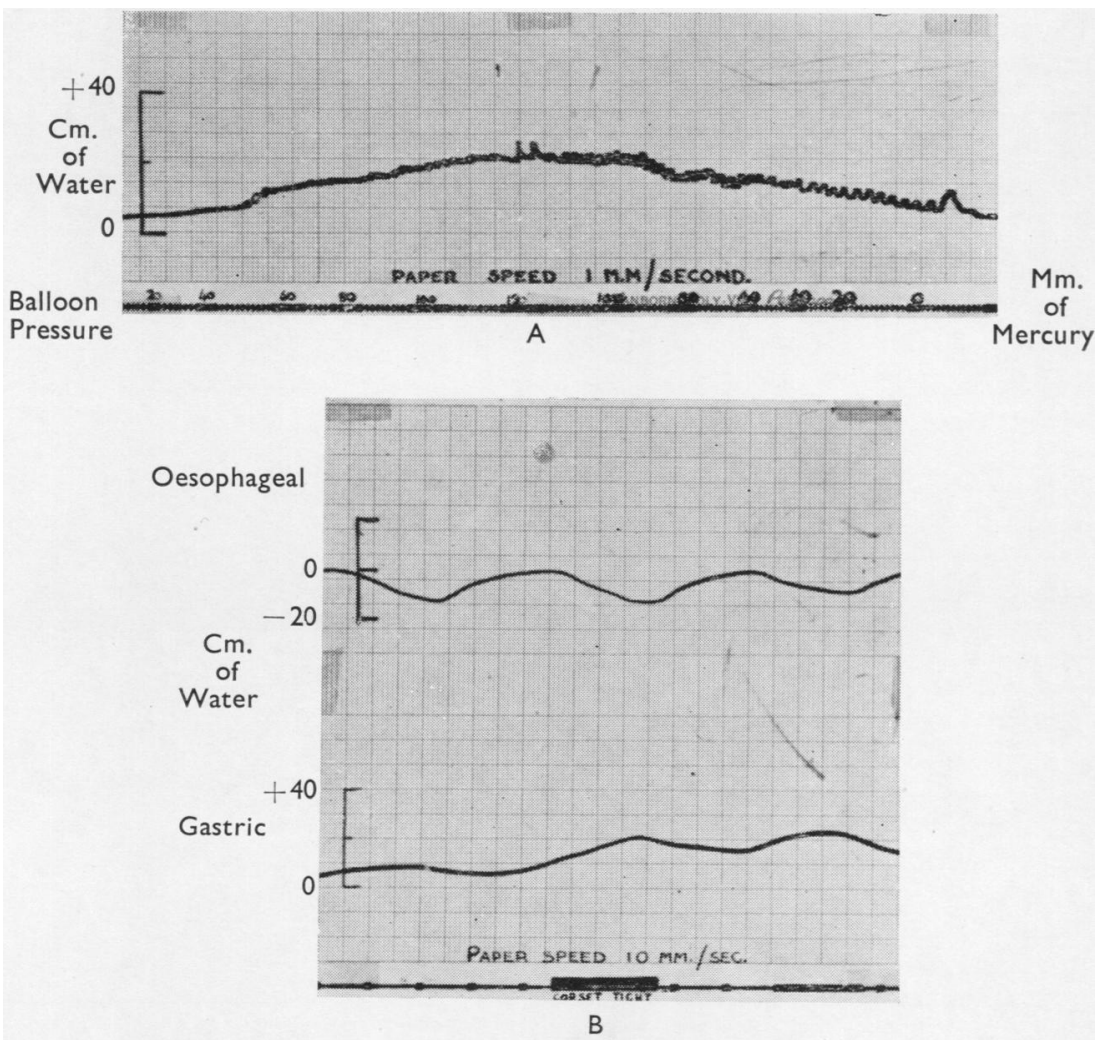

Fig.9.-Effect of external abdominal compression upon the intragastric pressure (see text). (A) Graduated increase of balloon pressure to $120 \mathrm{~cm}$. of water followed by graduated release. (B) Simultaneous intra-oesophageal and intragastric pressures recorded while a corset was suddenly tightened. 
Abdominal compression with a corset or belt increases the basal intraperitoneal pressure but has little effect upon the intrapleural pressure so that the pleuro-peritoneal gradient increases.

\section{INTRINSIC INFLUENCES UPON THE INIRAGASTRIC PRESSURE}

A catheter in the stomach will also register pressures generated by gastric tone and peristalsis. Quigley has shown that the pressures produced by gastric muscle tone are in the region of $0.5 \mathrm{~cm}$. of water. Such low pressures do not invalidate intragastric recordings as a measure of the intraperitoneal pressure. Gastric peristaltic waves produce higher pressures than these and the recorded wave is typically gradual in onset, slow in reaching a peak and slow in relaxing.

The stomach is capable of rapid contractions during vomiting and belching. A tracing, taken during a belch, shows waves which oscillate at a rate of 4 per second and which attain a pressure from 5 to $15 \mathrm{~cm}$. of water (Fig. 10). The pressure rise within the oesophagus starts $3 / 10$ of a second after that in the stomach, and, though the gastric pressure returns to basal level as soon as the eructation terminates, the oesophageal pressure drops more gradually to reach its base line three seconds later. In many other recordings it has been obvious that the subject strained to eructate $\frac{C}{0}$ causing a simultaneous pressure rise in stomach and oesophagus; but in the case illustrated the belch was emitted without extraneous encourage 0 ment. The time lag before the rise in oesophagear pressure is a measure of the time taken for the mass of air to pass from gastric to oesophageatcatheter tips. It would seem that the rise in gastriew pressure is, at least in part, due to an increase in gastric muscle tone whereas the waves produced while the belch is in progress are due to rapid gastric contractions. The transmission of thesev rapid pressure changes to the oesophagus is imme $\overrightarrow{00}$ diate, because the cardia is open so that a continuous air column extends from oesophagus to stomach. The interpretation of the delay in the drop of the intra-oesophageal pressure is that theD cardia has closed, the stomach has relaxed, but ai is still distending the oesophagus.

A gastric pressure tracing was also fortuitousl obtained while an unlucky student vomited. High pressures were produced and the stylus passed off the recording paper which was calibrated up to a pressure of $60 \mathrm{~cm}$. of water (Fig. 10). Abdomiogag

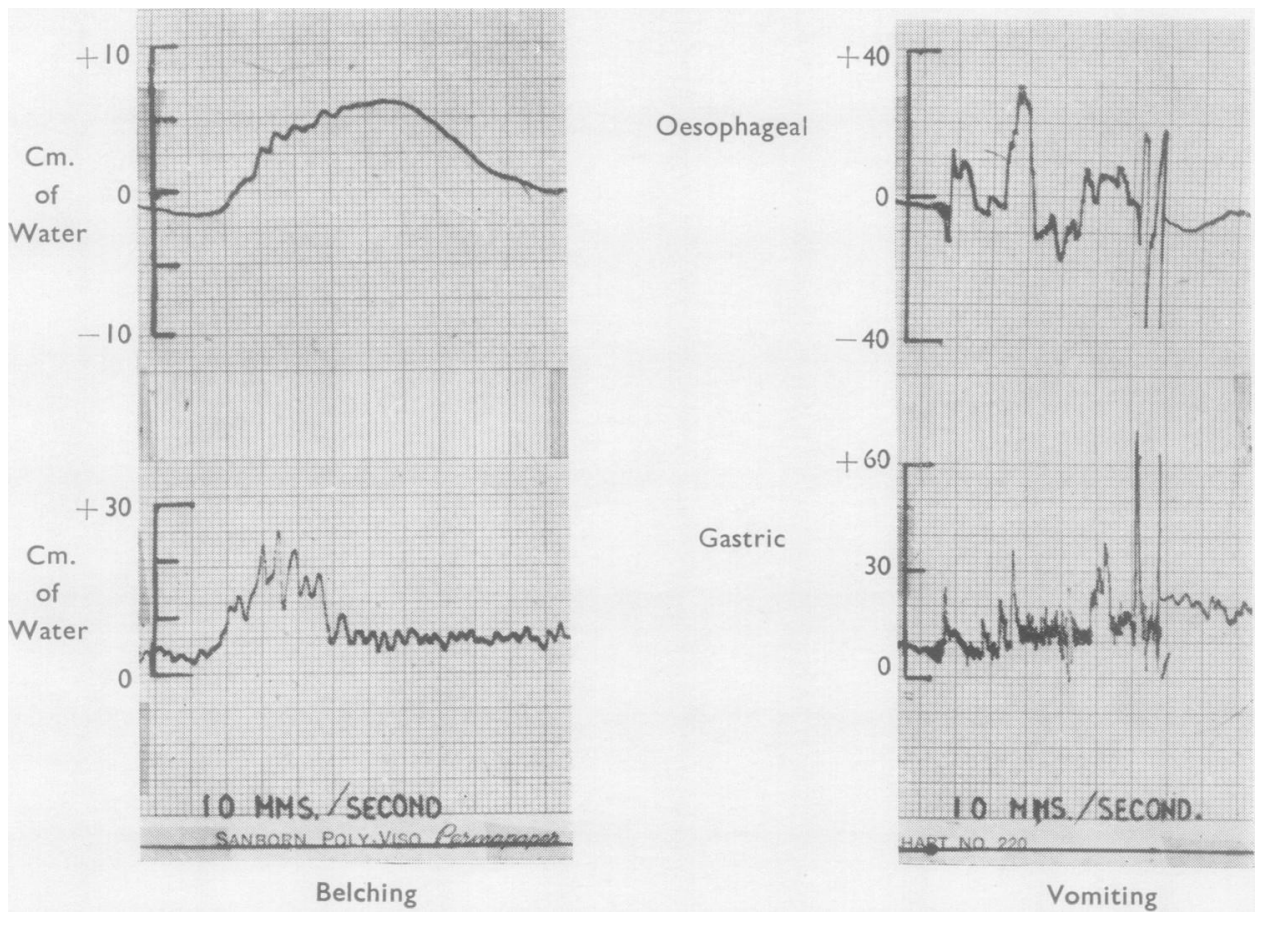

Fig. 10.-Simultaneous intra-oesophageal and intragastric pressures recorded during belching and vomiting. These tracings demonstrate that the stomach is capable of rapid contractions. 
muscle contraction was probably largely responsible for the high pressure, but rapid fluctuations similar to those produced by belching were recorded during the effort of retching.

A series of animal experiments have been done so as to measure gastric pressures in the presence of pyloric spasm. In the dog it is possible to cause pyloric spasm by forceful traction on the fundus of the gall bladder. During this manœuvre the intragastric pressure rises even in the absence of visible peristalsis (Fig. 11). This is probably an indication that a general increase in gastric tone accompanies pyloric spasm.

Finally an attempt has been made to estimate the effect of gastric distension upon the intragastric pressure. Certainly the basal intragastric pressure does rise when the stomach distends even though gastric mobility is inhibited by food

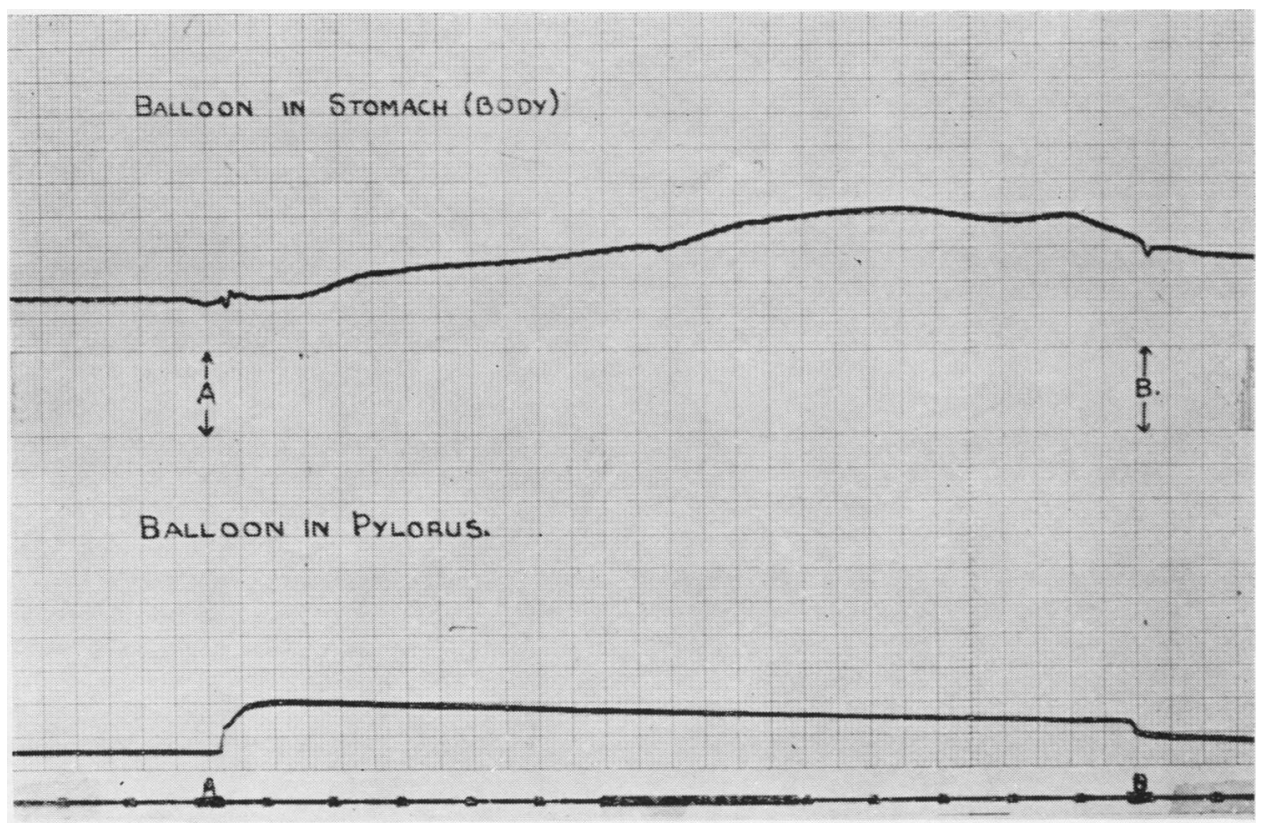

Fig. 11.-Simultaneous gastric and pyloric pressures recorded with intraluminal balloons while forceful traction was exerted on the gall bladder of an anaesthetized dog. Traction applied at A and released at B. (Paper speed, $10 \mathrm{~mm}$. $/ \mathrm{sec}$.)

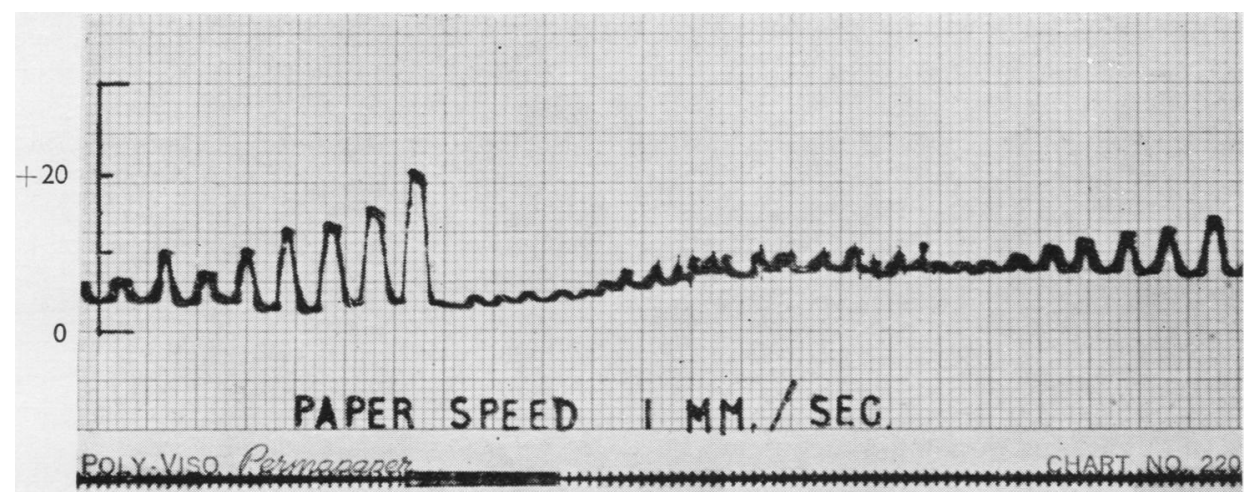

Seidlitz

Powder

FIG. 12.-Intragastric pressure tracing showing the effect of gastric distension upon the basal pressure. The second Seidlitz powder was taken by the subject while the continuous line on the time marker was being written. The subject graded the depth of respiration before and after gastric distension. 
(Cannon, 1911). Fig. 12 shows the effect upon the supine intragastric pressure after the stomach had been distended with Seidlitz powders.

The rise in pressure which accompanies gastric distension is said by Quigley to be due to reflex increase in gastric and bulbar tone. This is a possible explanation, but the increase in volume of the stomach within a packed peritoneal cavity may also affect the general abdominal pressure.

\section{Discussion}

Gastro-oesophageal Regurgitation. - The intragastric pressure is the force which produces gastro-oesophageal regurgitation. It has been shown that the pressure within the resting stomach is a measure of the general intraperitoneal pressure, and that significant pressure changes within it also result from its own muscular activity. The factors which influence the general peritoneal pressure have been shown to be:

(a) Posture.-With the body erect the pressure within the upper abdomen is low. Wilson and Irving (1931), using the balloon technique, reported sub-atmospheric pressures high in the gastric fundus, and this has been supported by Quigley (1944), who occasionally recorded pressures of $-8 \mathrm{~cm}$. of water immediately subphrenically with the subject erect. None of my recordings has shown subphrenic negativity, but the mean pressure is usually only slightly above zero. Because of the low pressure gradient across the diaphragm one would not expect gastro-oesophageal regurgitation to occur in the erect position, and, in fact, regurgitation is more facile when the subject lies down or bends forward. However, post-prandial regurgitation is not uncommon with the body erect, and it is then necessary to postulate that intrinsic causes of a raised intragastric pressure have precipitated reflux in spite of the subject's being upright.

(b) Respiration.-When considering the force which produces regurgitation from the stomach into the oesophagus, it is not sufficient to measure the intragastric pressure alone. The effective regurgitation force must be the difference between the gastric and the oesophageal pressures. The gastro-oesophageal "sphincter" mechanism is the bar to regurgitation and previously it has been shown that, in the absence of gross anatomical anomaly at this site, regurgitation may be precipitated simply as a result of an increase in the gastro-oesophageal pressure gradient (Marchand, 1955). This gradient is largely dependent upon respiration and during deep inspiration a considerable pressure differential may develop across the diaphragm $(100 \mathrm{~cm}$. of water). The significant dynamic effect of this is confirmed by clinical and radioscopic findings. During barium examinations of the oesophagus the bolus is seen to retained above the diaphragm whenever the sue ject inspires even though the oesophagus contracts sufficiently forcibly to produce a phrenic ampulla Also reflux of barium from the stomach occuss most readily on inspiration. Creamer (1955) hæ्ष shown graphically that with normal anatomicat conditions reflux occurs only during inspiration

(c) Coughing and Straining.-On superfici consideration it would seem that the forceful mus. cular action involved in defaecation, coughing, the Valsalva test, and straight leg lifting would terid to throw a greatly enhanced strain upon the gastro-oesophageal sphincter mechanism. Veq considerable intraperitoneal pressures are generated by such activities, but, so far as gastric reflux is concerned, the effect is largely cushioned by $\frac{\mathbb{Q}}{\mathbb{Q}}$ simultaneous rise in the intrapleural pressure. Indeed the effective regurgitation force is appre ciably less than that produced by postural changes and inspiration. It is my experience that the Vade salva test, coughing, and other straining efforts ak ineffective in producing gastro-oesophageal regui gitation in the normal individual. However, thes actions do increase the peritoneo-pleural gradirem (Figs. 7 and 8), probably as a result of forceff abdominal muscular contraction, and in the presence of an inefficient gastro-oesophageat "sphincter" they may precipitate reflux.

External compression of the abdomen exerts pronounced effect upon the intraperitoneal pres sure. It has been shown that corsets and other compressing garments raise the mean intro peritoneal pressure appreciably (Fig. 9) and the effects of posture, respiration, and straining are aggravated when the abdomen is rigidly confinee

The intragastric pressure is also influenced bo intrinsic factors. The experiments described have indicated that the mean intragastric pressure is raised in the presence of pyloric spasm (Fig. 18 presumably as a result of increased gastric tone Distension of the stomach is also accompanied bi a raised intraluminal pressure (Fig. 12) possibly due to a reactive increase in gastric tone and possibly to tighter " packing" of the abdominal cors tents. The high gastric pressures which acconte pany pyloric spasm and gastric distension go long way towards explaining regurgitation whieb occurs when the subject is erect. Belching afte्⿱一土 large meals is commonplace, whilst heartburfe following certain foods such as spices, onionsp and others which vary from individual to ind vidual, may be due to pyloric spasm following gastric irritation. In the presence of pylori 
spasm, sudden forceful gastric contractions, such as those recorded during belching (Fig. 10), may well raise the intragastric pressure to levels capable of overcoming the resistance of the gastrooesophageal "sphincter." This may explain the regurgitation dyspepsia which so often accompanies gall-bladder disease and duodenal ulcer.

In the absence of hiatus hernia, habitual flatulent dyspepsia occurs in several well-defined groups of people. They are the obese, the wellmuscled individual who has recently gained weight, the pregnant woman, the air-swallower, the glutton, and the person who cannot tolerate certain foods. In all these types of people one or more reasons for a high intragastric pressure are operative. The obese, the pregnant, and those with large abdominal tumours suffer because the packing of their abdominal contents results in a high basal intraperitoneal pressure which in turn enhances the adverse effects of posture, respiration, etc. In most instances a cure is effected by dieting, parturition, or surgical removal of the tumour. The air-swallower and the glutton suffer because of their distended stomachs, and in these cases both intrinsic and extrinsic (equivalent to an abdominal tumour) causes are operative. One often meets men in their thirtics who, having previously led a physically active life, have settled into the luxuries of domesticity and develop dyspepsia. It is not necessary to implicate the inexpert catering of a newly acquired wife: the opposite is the more common truth. Their abdominal muscles retain much of the tone of youth, but the abdominal contents have become packed with newly-acquired fat. The actual gain in weight need not necessarily be great, a few pounds judiciously placed in the omentum are enough to raise the general intraperitoneal pressure considerably in the presence of the splinting effect of strong abdominal walls. Cure awaits the unpleasantness of weight reduction or the inevitable onset of abdominal laxity, but in the meantime the oesophageal hiatus may have disrupted in the face of the inexorable abdominal pressure.

\section{Hiatus Hernia}

Congenital and traumatic diaphragmatic herniae may be compared with congenital umbilical and ventral herniae. In all these conditions abnormal defects exist through which some abdominal viscus is forced by the intraperitoneal pressure. Hiatus herniae and indirect inguinal herniae, on the other hand, occur through sites where normal structures traverse the abdominal boundaries. The inguinal mechanism, with its multilayered structure and the obliquity of its canal, is better adapted than the oesophageal hiatus to resist the intraperitoneal pressure. It is now widely believed that an indirect inguinal hernia is seldom caused by a breakdown of the defensive mechanism of the inguinal canal, but is usually due to the presence of a preformed congenital sac. It is tempting also to postulate this for hiatus hernia, but there is no convincing evidence to support such a belief. One must accept that infantile hiatus herniae are secondary to congenital hiatal weakness, but it is felt that the more common adult hernia is an acquired lesion in the sense that a rupture occurs through a once normal and competent hiatus. With adult herniae, it is unlikely that a single cause operates, but two fundamental factors applicable to all types of hernia must be considered, namely, the structural defect and the extruding force.

The Structural Defect.-The oesophageal hiatus is the one vulnerable diaphragmatic opening. It transmits the mobile terminal oesophagus which is loosely attached to its edges and which, when empty, does not tightly fill the opening. The normal hiatus is elliptical and this shape is largely maintained by the anterior decussating fibres of the hiatal muscles (Fig. 13). The posterior crural

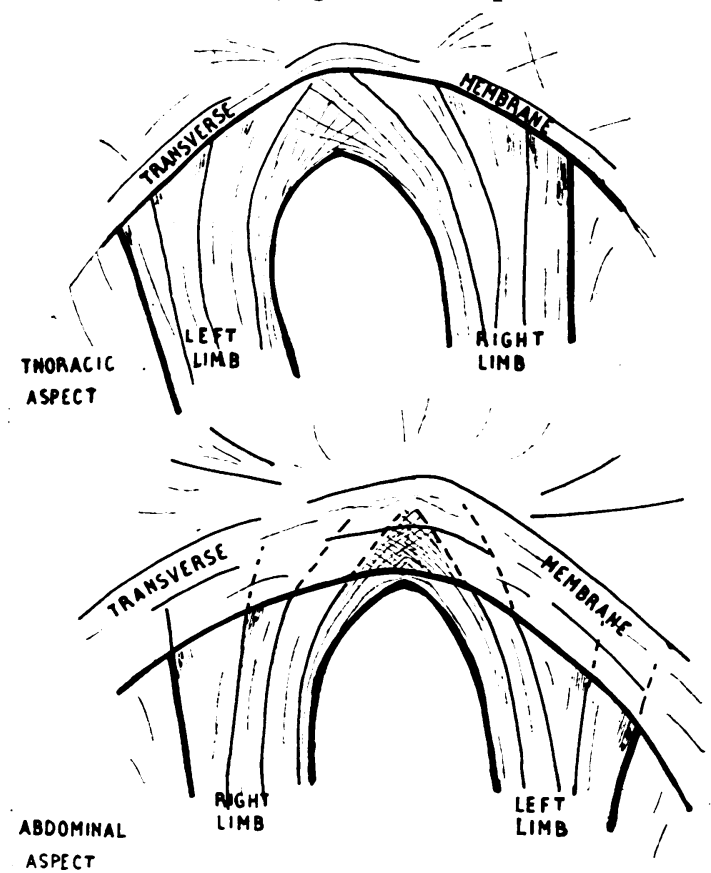

ASPECT

Fig. 13.-Diagram of the anterior hiatal muscles showing the decussation of the medially placed fibres. The anterior muscle is supported below by the transverse membrane of the central tendon of the diaphragm. 

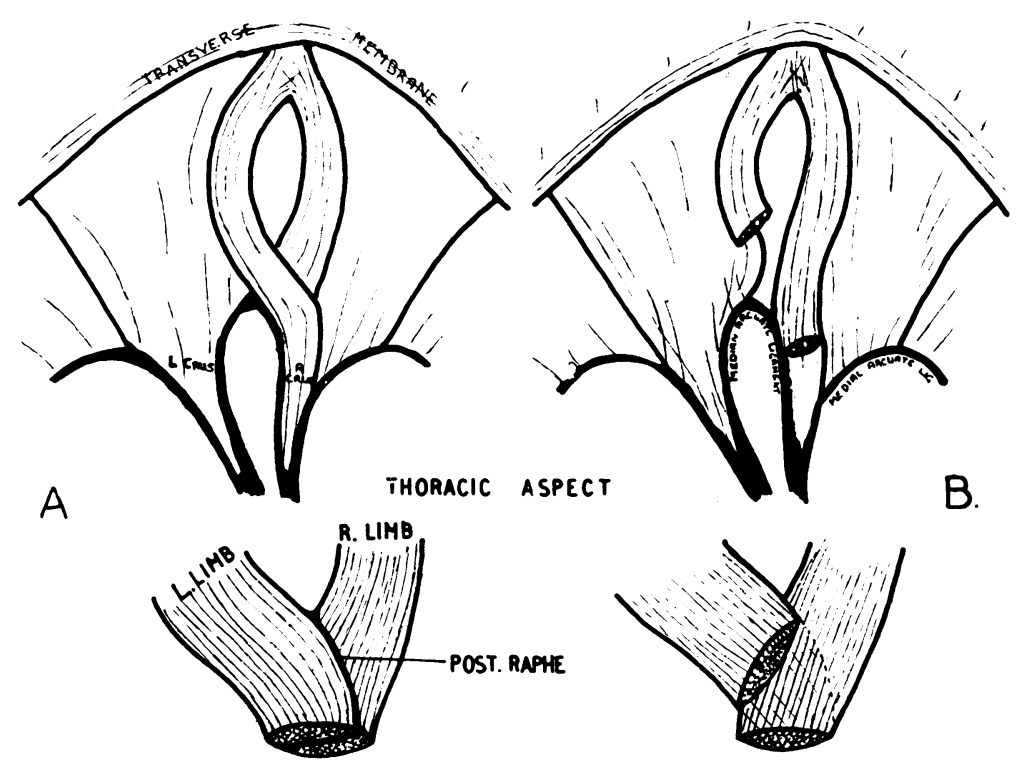

R. CRUS

FIG. 14.-Diagram illustrating the manner in which the right crural muscle bundles form hiatal margins of standard anatomical pattern.

limbs cross in a scissor-like fashion and the direction of their pull also tends to narrow the transverse diameter of the hiatus (Fig. 14). The posterior hiatal muscles are directly supported by the spine and the posterior half of the hiatal margin is extraperitoneal in situation. The anterior hiatal muscles, on the other hand, abut directly against the peritoneum ; they have no support on their thoracic aspect and receive little protection from the liver (Fig. 15). This, then, is the area which must bear the main brunt of the pressure exerted from below while distension of the oesophagus or other factors tending to widen the hiatus transversely throw additional strain upon these fibres (Fig. 16). Should they disrupt, the hiatus will assume a circular contour, its surface area will thereby be greatly increased and the support for the cardia and the peritoneal reflection will be lost. Once deprived of hiatal support the peritoneum will tend to bulge upward in front of the oesophagus and into the mediastinum. A second consequence of the loss of hiatal support is that the phreno-oesophageal ligament must now resist the full force of the intraperitoneal pressure, and should it stretch, as inevitably it must, the cardia will slide upwards into the mediastinum. A vicious cycle is initiated, for as more bulk occupies the opening, the posterior hiatal limbs, so lightly joined, are divaricated and further widening of the hiatus is allowed. The transverse ligament of the central tendon prevents the hiatus enlarging number of patients hofre been sent to us with a diagnosis of pure para oesophageal hernia, but on repeating the examig tion with sustained abdominal compression or Trendelenburg tilt the coexistence of an element迎 of slide has always been demonstrated. It is

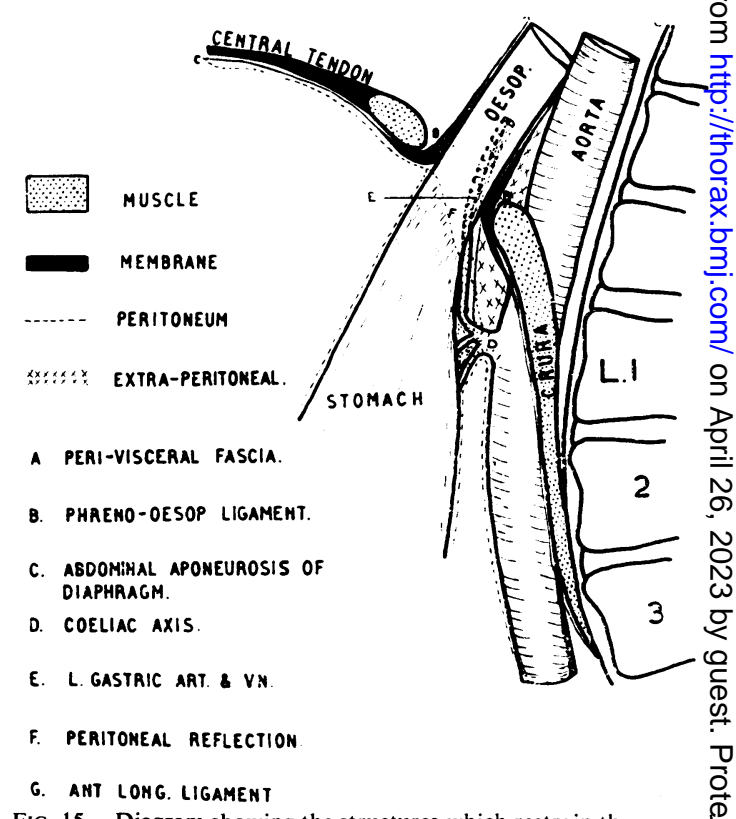

Fig. 15.-Diagram showing the structures which restrain the upward displacement of the cardia. (Note that only the anterior margin at the hiatus abut directly against peritoneum.)

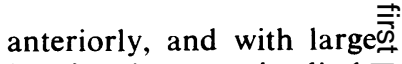
herniae the posterior limb? may become widely sepa $\frac{\overline{\bar{D}}}{\overline{\frac{\bar{T}}{5}}}$

The phreno-oesophagea $\mathbb{B}$ ligaments, the vagus nervesణ and the left gastric vessels may restrain the cardia to some extent and the an $\vec{\omega}$ terior surface of the funduge toneal sac in front of the oesophagus. In this wayt the appearances of a para $\vec{\circ}$
oesophageal hernia areo produced. A para-oeso은 phageal hernia is there fore a variant of the slide ing variety, and indeed $i \stackrel{\mathbb{P}}{\mathbb{P}}$ must be excessively rare for this type of hiatu最 hernia to be uncomplicated by some degree of upwarde slide of the cardia. A , 


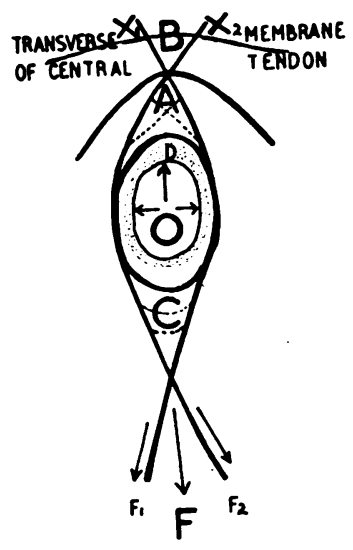

. DisRupting force = ImTRAPERITONEAL PRESSURE OESOPHAGEAL DISTENSION

RESTRAINING FORCES=

$X-F$ lines of force of crupal muscle tone and contraction

A decussation of anterior muscle fibaes

B tansverse membrane support to amterior fibres

C posterior intea-tendinous fibres

OESOPHAGO-PHRENIC LIGAMENT

FIG. 16.-Diagram illustrating the forces which maintain the elliptical shape of the hiatus and those which tend to disrupt it.

descriptively accurate to call this variety of hiatus hernia a rolling hernia, but it is not justifiable to categorize it, as Sweet (1952) does, as a pathological entity separate from the sliding variety.

Since the time of Akerlund (1926) it has been said that ageing processes affect the hiatus adversely, and Schatzki (1932) has estimated that the hiatus is abnormally large in $75 \%$ of people over the age of 60 . It is therefore not unreasonable to postulate that degenerative changes of the collagen matrix may weaken the anterior hiatal fibres. Degenerative changes due to age alone cannot always be implicated, and inherent individual structural weaknesses or softening of the collagen elements of the hiatus during pregnancy may predispose to the development of a hernia in the face of the stress of the abdominal pressure.

The EXTRuding Force.-At the inguinal region the pressure gradient is between the peritoneum and the atmosphere, but at the hiatus the gradient is between the abdomen and the chest. In the horizontal position, therefore, the net force exerted on the hiatus is greater than that pressing on the inguinal region, and with deep inspiration the pressure may be considerable. However, on exertion the intrapleural pressure rises above atmospheric and the strain exerted on the diaphragm from below is partly cushioned above. When the body is erect the force acting against the diaphragm is much less than that exerted on the groin, for the abdominal organs "fall " away from the hiatus and the pressure is greatest in the pelvis.

The effects of corsets, tight clothing, belts, and obesity are felt at both regions. Abdominal compression raises the intraperitoneal pressure markedly but does not affect the intrapleural pressures. Adiposity, pregnancy, and abdominal tumours increase the intraperitoneal pressure by virtue of the greater weight and the greater volume of the contents. In such circumstances the elastic abdominal wall will stretch, but this may not entirely accommodate for the increased bulk of the contents, with the result that the viscera are tightly "packed" and the general intraperitoneal pressure rises. The same forces which tend to initiate gastro-oesophageal reflux also throw a strain upon the anterior poorly protected and poorly supported fibres of the hiatal mechanism. Donnelly (1953) was so struck by the long history of flatulent dyspepsia which preceded the discovery of a hiatus hernia in many of his cases that he postulated that regurgitation itself weakened the hiatal mechanism. This may be so, but it seems more reasonable to believe that the same stress which produces regurgitation also in time causes disruption of the anterior hiatal muscles and so starts the first change in a vicious cycle which results eventually in the development of hiatus hernia.

\section{SUMMARY}

An attempt has been made to measure the pleural and peritoneal pressures which exert a strain upon the diaphragm. The object has been to study the possible part these may play in the pathogenesis of gastro-oesophageal regurgitation and hiatus hernia.

The pressure within the resting stomach has been shown to be a measure of the general intraperitoneal pressure.

The effects of posture, respiration, forcible straining, and external abdominal compression upon the intraperitoneal pressure and the pressure gradient across the diaphragm have been measured.

Intrinsic causes of a raised intragastric pressure have been analysed.

The importance of all these factors in the production of reflux dyspepsia and in the development of hiatus hernia is discussed.

I must thank the Council for Scientific and Industrial Research of South Africa, who supplied me with the equipment necessary to conduct the experi- 
ments reported here. Mr. D. Smith, of the Department of Surgery, University of the Witwatersrand, has maintained and operated the electromanometer and is responsible for the photographic reproductions of the recordings. Mr. L. Fatti, Head of the Department of Thoracic Surgery, has advised and encouraged me in all aspects of my interest in hiatus hernia.

\section{REFERENCES}

Åkerlund, A. (1926). Acta Radiol. (Stockh.), 6, 63.

Allison, P. R. (1948). Thorax, 3, 20.

- (1951). Surg. Gynec. Obstet., 92, 419.

Appleton, A. B., Hamilton, W. J., and Simon, G. (1946). Surface and Radiological Anatomy, 2 nd ed. Heffer, Cambridge.

Barrett, N. R. (1954). Brit. J. Surg., 42, 231.

Cannon, W. B. (1911). The Mechanical Factors of Digestion. Arnold, London.

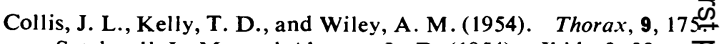
Satchwell, L. M., and Abrams, L. D. (1954). Ibid., 9, 22. Creamer, B. (1955). Lancet, 1, 279.

Donnelly, B. (1953). Brit. J. Radiol., 26, 441.

Dornhorst, A. C., and Leathart, G. L. (1952). Lancet, 2, 169.

Harrington, S. W. (1952). Rev. Gastroent., 18, 243.

Lam, C. R. (1939). Arch. Surg. (Chicago), 39, 1006.

Lerche, W. (1950). The Esophagus and Pharynx in Action. Thomaş Springfield, Illinois.

Marchand, P. (1952). Brit. J. Radiol., 25, 476.

(1955). Brit. J. Surg., 42, 504.

Peters, P. M. (1955). Thorax, 10, 27.

Quigley, J. P. (1944). In Medical Physics, Vol. 1, p. 313, ed. Glasse. O. Year Book Publishers, Chicago.

Schatzki, R. (1932). Fortschr. Röntgenstr., 45, 177.

Sweet, R. H. (1952). Ann. Surg., 135, 1.

Wade, O. L., and Gilson, J. C. (1951). Thorax, 6, 103.

Wilson, M. J., and Irving, L. (1931). Canad. med. Ass. J., 25, 658 